ARTICLE

DOI: $10.1038 / \mathrm{s} 41467-017-01590-5$

\title{
The genomic landscape of pediatric myelodysplastic syndromes
}

Jason R. Schwartz ${ }^{1}$, Jing Ma², Tamara Lamprecht ${ }^{2}$, Michael Walsh², Shuoguo Wang ${ }^{3}$, Victoria Bryant ${ }^{2}$, Guangchun Song ${ }^{2}$, Gang Wu (10 ${ }^{3}$, John Easton ${ }^{3}$, Chimene Kesserwan ${ }^{1}$, Kim E. Nichols ${ }^{1}$, Charles G. Mullighan (1) ${ }^{2}$, Raul C. Ribeiro ${ }^{1} \&$ Jeffery M. Klco (i) ${ }^{2}$

Myelodysplastic syndromes (MDS) are uncommon in children and have a poor prognosis. In contrast to adult MDS, little is known about the genomic landscape of pediatric MDS. Here, we describe the somatic and germline changes of pediatric MDS using whole exome sequencing, targeted amplicon sequencing, and/or RNA-sequencing of 46 pediatric primary MDS patients. Our data show that, in contrast to adult MDS, Ras/MAPK pathway mutations are common in pediatric MDS (45\% of primary cohort), while mutations in RNA splicing genes are rare ( $2 \%$ of primary cohort). Surprisingly, germline variants in SAMD9 or SAMD9L were present in $17 \%$ of primary MDS patients, and these variants were routinely lost in the tumor cells by chromosomal deletions (e.g., monosomy 7) or copy number neutral loss of heterozygosity $(\mathrm{CN}-\mathrm{LOH})$. Our data confirm that adult and pediatric MDS are separate diseases with disparate mechanisms, and that SAMD9/SAMD9L mutations represent a new class of MDS predisposition.

\footnotetext{
${ }^{1}$ Department of Oncology, St. Jude Children's Research Hospital, 262 Danny Thomas Place, Mail Stop 260, Memphis, TN 38105, USA. ${ }^{2}$ Department of Pathology, St. Jude Children's Research Hospital, 262 Danny Thomas Place, Mail Stop 342, Memphis, TN 38105, USA. ${ }^{3}$ Department of Computational Biology, St. Jude Children's Research Hospital, 262 Danny Thomas Place, Mail Stop 1135, Memphis, TN 38105, USA. Jason R. Schwartz and Jing Ma contributed equally to this work. Correspondence and requests for materials should be addressed to J.M.K. (email: jeffery.klco@stjude.org)
} 
M yelodysplastic syndromes account for $<5 \%$ of pediatric hematologic malignancies with an incidence of 2-4 cases/million ${ }^{1}$. The prognosis of pediatric MDS is typically poor, because cytotoxic chemotherapies like those used to treat acute leukemia are not successful, thus leaving only bone marrow transplantation as a curative option ${ }^{1,2}$. Much has been learned about adult MDS through next-generation DNA sequencing. Multiple large cohort studies of adult MDS patients found recurrent mutations in genes important in epigenetic regulation (e.g., TET2, ASXL1, and DNMT3A), and RNA splicing (e.g., SF3B1 and U2AF1) ${ }^{3-7}$. However, in limited studies on pediatric MDS, mutations in these genes are uncommon ${ }^{8,9}$. This is not surprising as there are well-accepted clinical and morphologic differences between pediatric and adult MDS (e.g., bone marrow hypocellularity is more common in children) leading the World Health Organization (WHO) to classify MDS differently in adults and in children ${ }^{10}$.

It is becoming increasingly recognized that germline variants in different transcription factors, such as GATA2, RUNX1, ETV6, or $C E B P A$, can lead to familial MDS/AML ${ }^{11-14}$. In particular, germline GATA2 variants have been shown to occur in $7 \%$ of pediatric primary $\mathrm{MDS}^{15}$. In contrast, other studies using targeted sequencing of children with idiopathic bone marrow failure or MDS found pathogenic variants in only approximately $10 \%$ of patients ${ }^{16}$, suggesting the need for more comprehensive sequencing. The spectrum of genes harboring germline variants in pediatric MDS has also recently begun to expand beyond transcription factors, including ANKRD26 $6^{17}$ and $S R P 72^{18}$. Recently, germline variants in SAMD9 and SAMD9L have been reported in clinical syndromes affecting multiple organ systems that are also associated with MDS and monosomy $7^{19-22}$, as well as isolated familial MDS ${ }^{23}$.

Despite this progress, no study to date has performed comprehensive sequencing on a pediatric MDS cohort to fully understand somatic and germline variation in this neoplasm. In this study we perform tumor and normal whole exome sequencing (WES) on 32 pediatric primary MDS patients and targeted sequencing on another 14 cases through a single institution study focused on defining the genomic landscape of pediatric MDS. For comparison, we similarly characterize 23 cases with overlapping features of MDS and myeloproliferative neoplasm (MDS/MPN), namely juvenile myelomonocytic leukemia (JMML), and 8 cases of AML with myelodysplasiarelated changes (AML-MRC). We show that Ras/MAPK pathway mutations are common in pediatric primary MDS (45\%) while mutations in RNA splicing genes are rare (2\%), and that germline SAMD9/SAMD9L mutations are present in 17\% of primary MDS patients. These data suggest that pediatric MDS is separate from adult MDS with disparate underlying mechanisms.

\section{Results}

Sequencing of pediatric MDS samples. We performed next generation sequencing on a cohort of 77 pediatric patients with diagnoses of primary MDS $(n=46)$, MDS/MPN $(n=23,19$ of which were JMML), and AML-MRC $(n=8)$ (Fig. 1, Supplementary Data \#1 and Supplementary Fig. 1). Patients with a confirmed bone marrow failure syndrome, Down syndrome, or therapy-related MDS were excluded from this cohort. Three siblings with MDS that we recently described were included ${ }^{23}$. Of the primary MDS patients, $50 \%$ were classified as refractory cytopenia of childhood (RCC) and 50\% as refractory anemia with excess blasts (RAEB). Paired tumor-normal WES was performed on 54 of these cases with a median coverage of $\times 96$ in the normal and $\times 92$ in the tumor (Table 1, Supplementary Datas \#2 and 3). A subset of the variants was then validated by amplicon a

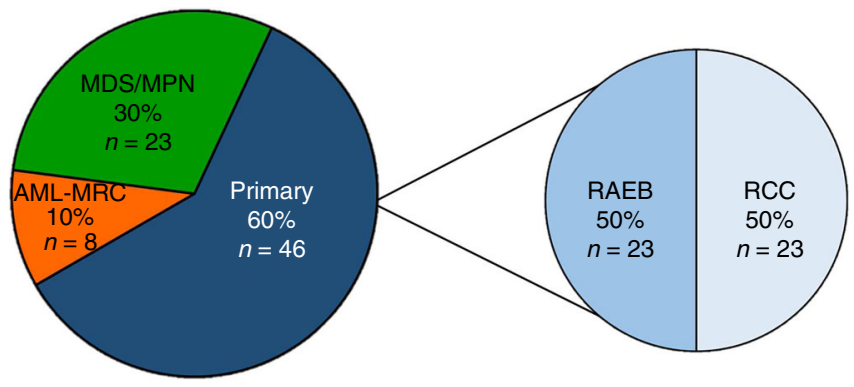

b

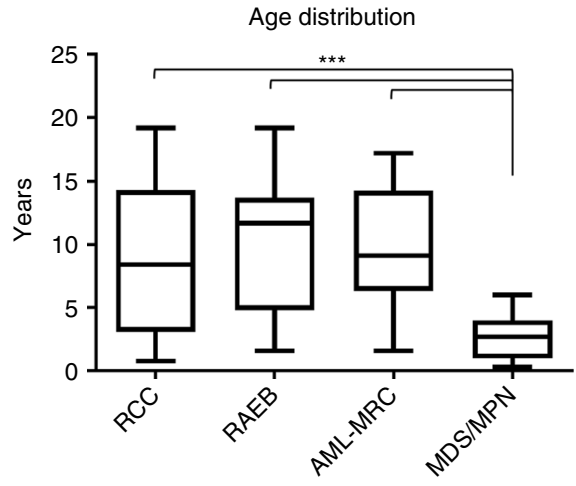

Fig. 1 Pediatric MDS cohort. a Pie charts depicting the distribution of the three diagnostic categories and subcategories of the pediatric MDS cohort; Total: $n=77$. b Distribution of age (in years) at diagnosis for the pediatric MDS cohort. Lines within boxes represent median age (RCC: 8.4, RAEB: 11.7, AML-MRC: 9.1, MDS/MPN: 2.7) and whiskers represent maximum and minimum. ${ }^{\star \star \star}: p<0.0001$ (student's $t$-test). AML-MRC AML with myelodysplasia-related changes, RAEB, Refractory anemia with excess blasts; RCC, Refractory cytopenia of childhood

sequencing to a mean depth of $\times 7000 . \mathrm{CD} 3+$ or unfractionated lymphocytes were flow sorted from the diagnostic sample to use as the source of normal comparator genomic DNA (gDNA) as other sources of normal gDNA were not available (Supplementary Fig. 2). This strategy has been successfully used in the past for JMML ${ }^{24}$ and other myeloid neoplasms ${ }^{25}$. Tumor-only material was available for the remainder of the cohort $(n=23)$, which was sequenced using a custom amplicon strategy targeting recurrent mutations identified in the WES cohort and other hotspots from adult and pediatric myeloid neoplasms with a median coverage of $\times 3000$ (Supplementary Datas \#4-6). In addition, RNA-sequencing was performed on 43 cases with available high-quality RNA (mean mapped reads: 107 million). For the 54 cases with WES, the somatic mutation rate and allele burden varied between the disease types (Fig. 2a, Supplementary Fig. 3) with the lowest number of mutations observed in RCC (mean: 4; range: $0-11$ ). Out of the 18 RCC cases and 14 RAEB cases sequenced by WES, 3 RCC cases and 1 RAEB case demonstrated no somatic coding mutations, 2 of which contained potential causative germline variants (see below). Furthermore, our data show that mutations in genes important in the Ras/ Mitogen-activated protein kinase (MAPK) pathway are the most common in our cohort while mutations in genes involved in RNA splicing are rare (Fig. 2b). Several other patients have no somatic mutations in genes typically implicated in myeloid neoplasms (Fig. 2c). The mutational signature for these cases was similar to that previously observed for AML (Signature 1A/1B), characterized by prominence of $\mathrm{C}>\mathrm{T}$ substitutions at $\mathrm{NpCpG}$ trinucleotides (Supplementary Fig. 4$)^{26}$. 
a

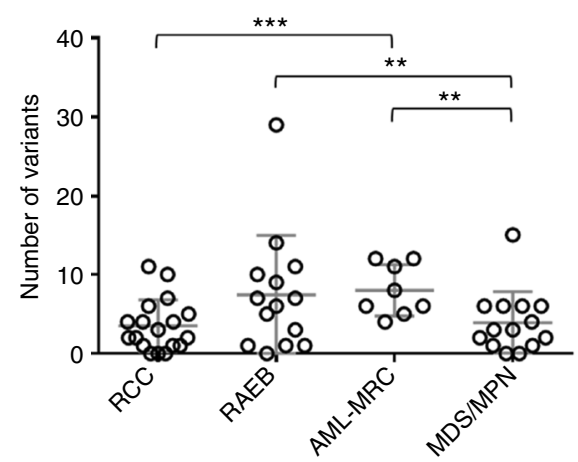

b

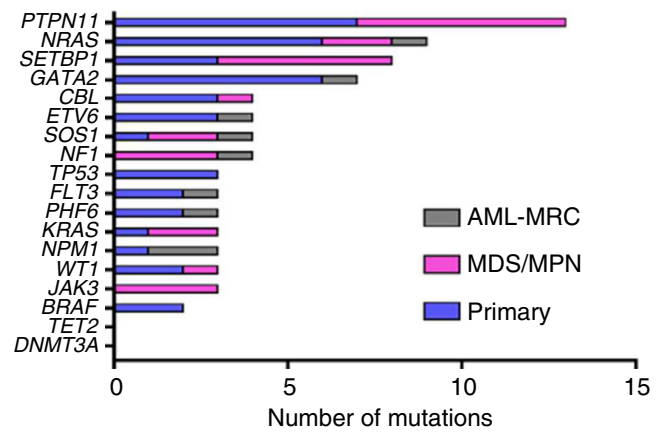

C

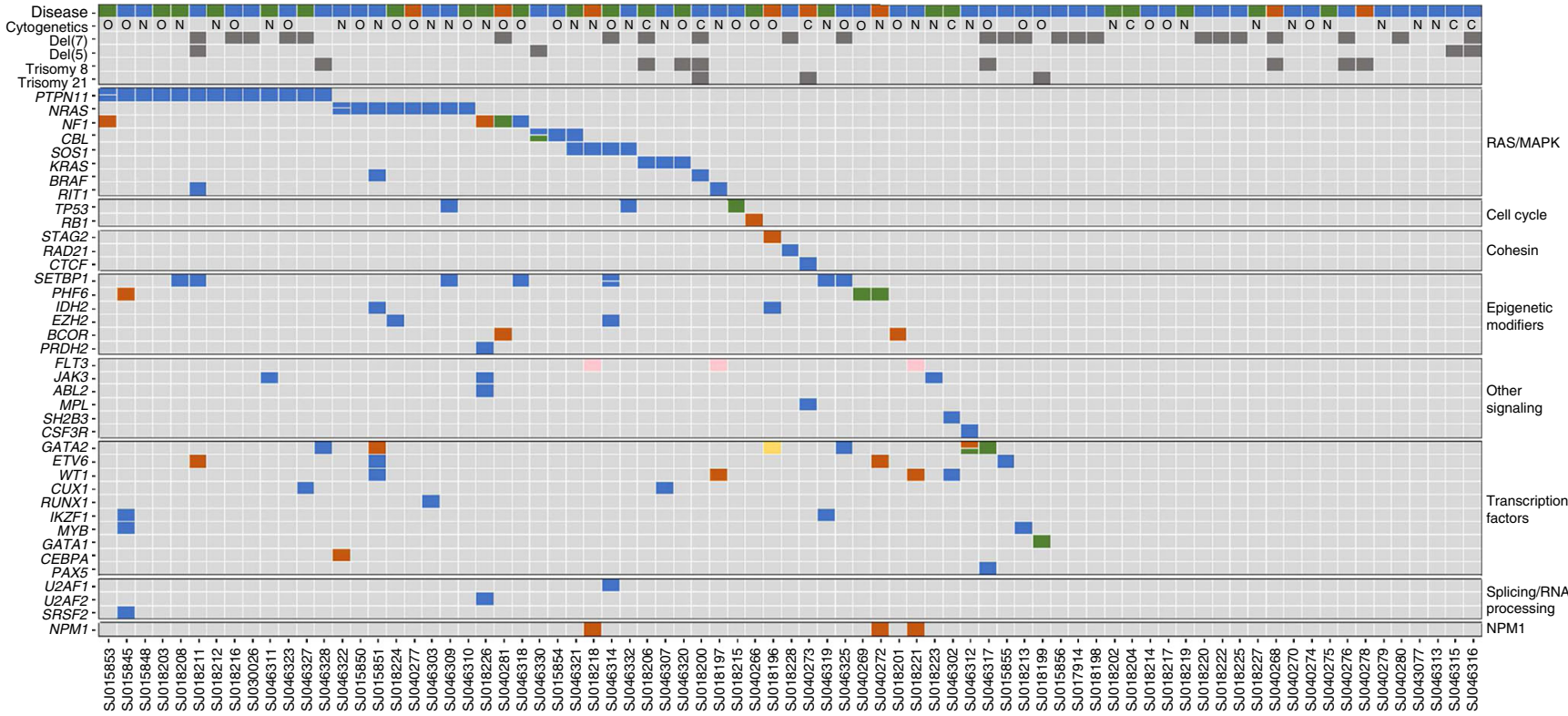

Mutation types $\square$ Missense $\square$ Nonsense $\square$ Frameshift $\square$ Indel $\square$ ITD

Disease $\square$ Primary MDS $\square$ MDS/MPN $\square A M L-M R C$

Fig. 2 Somatic mutations in pediatric MDS and related neoplasms. a Total number of somatic variants per patient in the 54 patients with WES data (includes silent, nonsense, missense, frame shifts, indels, ITD, and mutations within $3^{\prime}$ and $5^{\prime}$ UTR). ${ }^{* \star}: p=0.02 i^{\star \star \star}: p=0.003$ (student's t-test).

b The most common genes with somatic mutations in the full cohort of 77 patients (includes WES and targeted amplicon sequencing). Only somatic mutations with presumed functional consequences are shown. $\mathbf{c}$ Heat map showing the somatic mutational profile of the pediatric MDS cohort separated by gene functional groups. Only somatic mutations with presumed functional consequences are shown. Split cells indicate multiple mutations. O, other karyotype findings not listed separately; C, complex karyotype; N, normal karyotype

Copy number alterations in pediatric MDS. Copy number information, obtained from WES data and conventional karyotyping, determined that deletions involving chromosome 7 were more frequent in primary MDS $(n=19,41 \%)$ than in MDS/ MPN $(n=3,13 \%)$ and AML-MRC $(n=2,25 \%)$ (Fig. 3). Approximately $60 \%$ of RCC cases had deletions involving chromosome 7 (13 of 23), compared to only $26 \%$ of RAEB cases ( 6 of $23)$. Deletions involving chromosome 5 were infrequent $(n=4$, $5 \%)$; all occurrences were in RAEB cases. Trisomy of chromosomes $8(n=8,10 \%)$ and $21(n=3,4 \%)$, and deletions or loss of heterozygosity of $17(n=2,3 \%)$ were present at low frequency within the total cohort. In total, we detected 17 additional copy number abnormalities (including 3 cryptic chromosome 7 abnormalities) with WES that were not reported by standard conventional karyotyping with an average size of $40 \mathrm{Mbp}$ (Range: 0.02-159 Mbp) (Supplementary Data \#7). Alternatively, subclonal copy number alterations identified by conventional karyotyping $(n=12)$ were not found with WES. Notably, in 2 cases, subclonal del(7) was identified in 3 of 20 metaphases analyzed, yet was undetectable in the WES data. Copy number neutral events were identified in the lymphocytes (source of germline) in 3 patients, with involvement of chromosome 7 (x2) or 17 (Supplementary Fig. 5). The 7q CN-LOH event in SJ18228 has been previously reported by our group ${ }^{23}$.

Putative germline variants in pediatric MDS. We analyzed the 54 tumor-normal pairs for the presence of germline variants to determine if any cases harbored pathogenic variants that may predispose patients to a myeloid malignancy. It is important to note that our normal sample is from flow cytometry purified lymphocytes and thus cannot be used to definitively categorize a variant as germline given that it is possible for somatic mutations to arise in progenitor compartments affecting both myeloid and lymphoid cells, as has been shown in $\mathrm{JMML}^{27,28}$. We first analyzed all coding variants in over 1000 genes that have been implicated in pediatric cancer predisposition ${ }^{29}, \mathrm{JMML}^{30}$, familial 

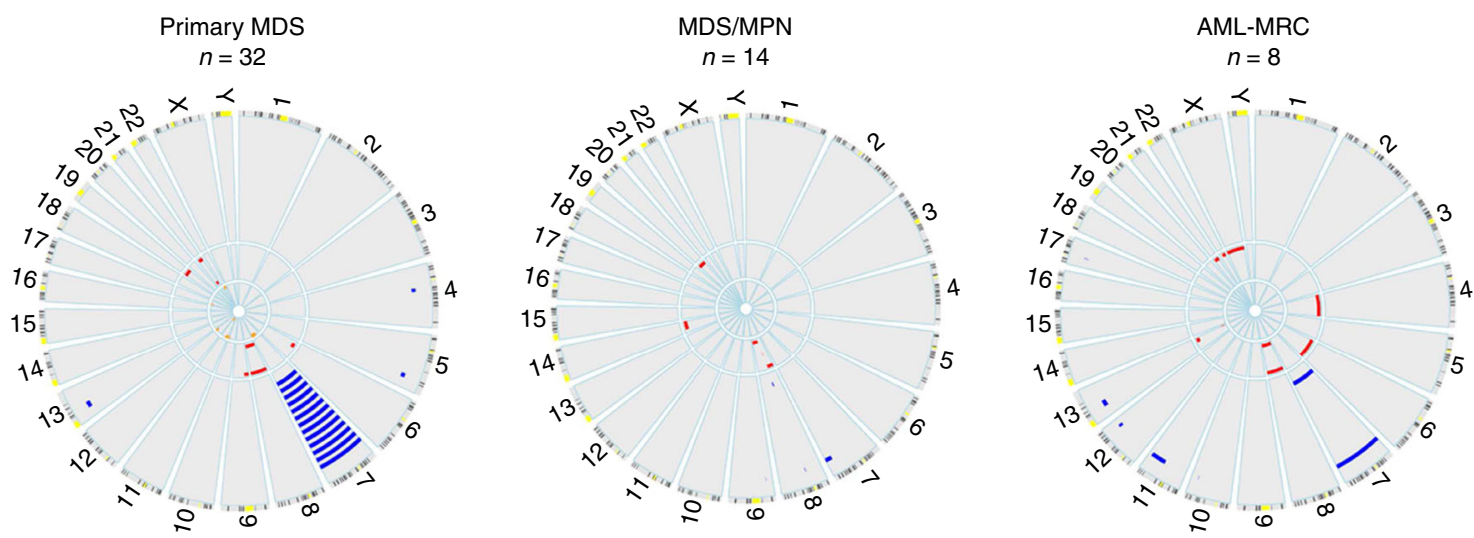

Fig. 3 Copy number analysis. Circos plots showing copy number alterations found with WES analysis of 54 tumor-normal pairs (events identified by conventional karyotyping are not included in these plots). Circumferential numbers indicate chromosome number, blue lines (outside ring) $=$ deletions, red lines (middle ring) = amplifications, and orange lines (inside ring) = copy number neutral-loss of heterozygosity

\begin{tabular}{|c|c|c|c|}
\hline & Total & DNA Seq. Technique & RNA Seq. \\
\hline \multicolumn{4}{|c|}{ Tumor/Normal Pairs $(n=54)$} \\
\hline Primary MDS & 32 & WES & 25 \\
\hline MDS/MPN & 14 & WES & 12 \\
\hline AML-MRC & 8 & WES & 6 \\
\hline \multicolumn{4}{|c|}{ Tumor Only $(n=23)$} \\
\hline Primary MDS & 14 & TSCA & Not Done \\
\hline MDS/MPN & 9 & TSCA & Not Done \\
\hline AML-MRC & 0 & TSCA & Not Done \\
\hline Total & 77 & & 43 \\
\hline
\end{tabular}

TSCA, TruSeq Custom Amplicon; WES, Whole-exome sequencing

$\mathrm{MDS} / \mathrm{AML}^{31}$, and adult MDS/AML ${ }^{32}$ (Supplementary Datas \#8 and 9) and a subset of these variants were classified according to the American College of Medical Genetics (ACMG) criteria ${ }^{33}$. In addition, we reported all loss of function variants (frameshift, nonsense and splice site alterations) in all genes (Supplementary Data \#10). In this study we used a VAF cut off of $>40 \%$ in the lymphocytes to classify variants as germline, unless there was evidence of $\mathrm{CN}-\mathrm{LOH}$ in the lymphocytes. We observed putative germline variants in PTPN11, NF1, and NRAS in patients with JMML, which are known associations ${ }^{30}$. Eight patients had germline variants in SAMD9 or SAMD9L (discussed below). Other potentially causative germline variants identified were in RRAS and BRCA2 (Table 2 and Supplementary Data \#9). The RRAS p.Q87L variant, which has been previously shown to increase MAPK activity and inhibit apoptosis ${ }^{34,35}$, has been reported in rapidly progressive JMML, and other germline variants in RRAS have been identified in Noonan Syndrome and $\mathrm{JMML}^{36}$. Only one patient had a presumed GATA2 germline variant (p.L375F). Resequencing of intron 4 of GATA2, a previously described mutation hotspot in pediatric $\mathrm{MDS}^{15}$, in all cases of primary MDS subjected to WES did not identify any additional germline events in these 32 cases. Of note, in the 14 primary cases with only tumor material available, there were 2 cases with GATA2 mutations at a variant allele frequency (VAF) of $>40 \%$, suggesting that they may be germline events (Supplementary Fig. 6). One of these cases had two separate GATA2 variants at a VAF $>40 \%$. In addition, we identified 2 PTPN11 variants via targeted amplicon sequencing with VAF's suggestive of germline lesions (Supplementary Fig. 7). Although material for normal comparator gDNA was not available to confirm germline lesions in these patients, the variants with VAF's $>40 \%$ were significantly higher than other somatic mutations present in the same patient.

Germline SAMD9 and SAMD9L variants are frequent in primary MDS. We previously described a germline SAMD9 variant (p.E1136Q) in three siblings with isolated familial $\mathrm{MDS}^{23}$, and others have described SAMD9 and SAMD9L variants as causative lesions in MIRAGE syndrome ${ }^{19,20}$ and Ataxia-Pancytopenia Syndrome (APS) ${ }^{21}$ or a syndrome resembling APS but with less severe neurological manifestations ${ }^{22}$, respectively. In total (including the previously reported three siblings), eight patients $(17 \%)$ in our primary MDS cohort had presumed SAMD9 $(n=4)$ or SAMD9L $(n=4)$ germline variants (RCC: 7, RAEB: 1$)$. Within the primary MDS cohort, $42 \%$ of the patients with loss of material on chromosome 7 have germline SAMD9 or SAMD9L variants. With the exception of the SAMD9 p.E1136Q variant, all variants identified were previously unreported missense variants (SAMD9: p.T778I; SAMD9L: p.W1180R, p.S626L $(n=2)$, and p.R1281K) and have variable predicted impacts on protein function (Fig. 4a and Supplementary Data \#11). The two patients (SJ018222 and SJ018225) with the SAMD9L p.S626L variants are relatives and culture of bone marrow fibroblasts from SJ018222 confirmed the germline status of this variant. Similar to previous studies evaluating $S A M D 9$ and $S A M D 9 L$ variants, all variants showed gainof-function activity that leads to decreased cell proliferation (Fig. 4b, c, and Supplementary Fig. 8). Furthermore, SAMD9/ SAMD9L gain-of-function variants inhibit the induction of ERK phosphorylation in response to serum (Supplementary Fig. 9). Depending on the extent of monosomy 7 observed in the tumor cells, the VAF of the SAMD9/SAMD9L variant decreased accordingly, suggesting a preferential loss of the allele harboring the deleterious variant (Fig. 4d). Previous studies showed the existence of acquired mutations in $S A M D 9$ or $S A M D 9 L$ that can serve to rescue the deleterious effects of a gain-of-function variant. Further, we previously reported on similar mechanisms in the included family with the germline p.E1136Q SAMD9 variant $^{23}$. In the remaining five patients, we observed a subclonal $\mathrm{CN}-\mathrm{LOH}$ event in the lymphocytes of SJ018225 that removes the pathologic p.S626L variant (see Supplementary Fig. 5). No other clear rescue mutations were observed in the remaining four patients.

Ras-MAPK pathway mutations are most common in pediatric MDS. Genes involved in the Ras/MAPK pathway were the most common mutations (including both germline and/or somatic) in 

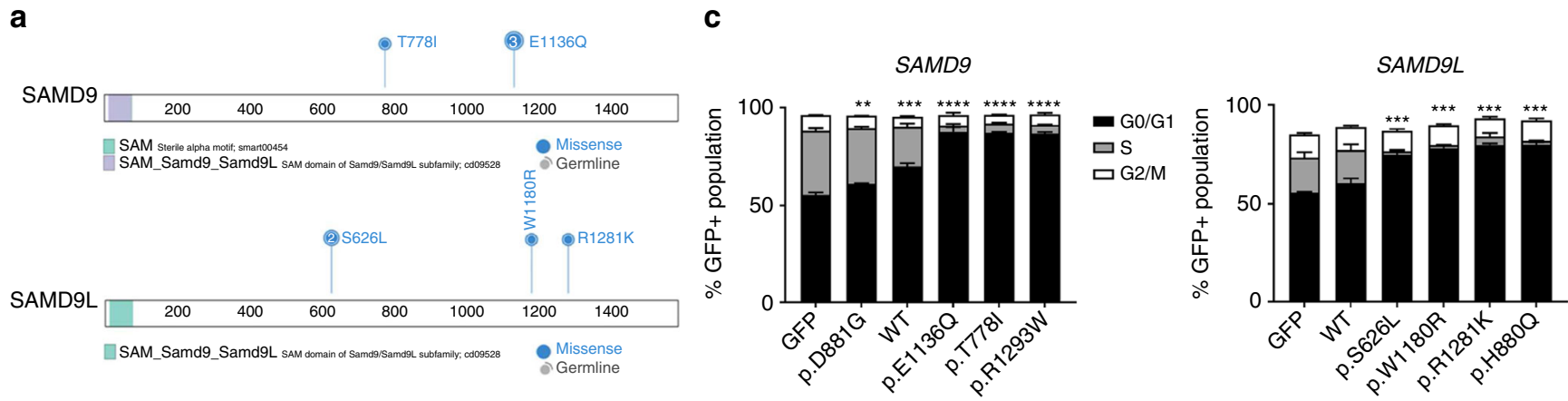

b

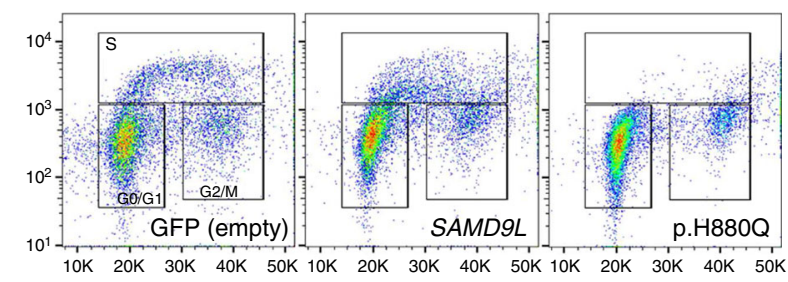

d
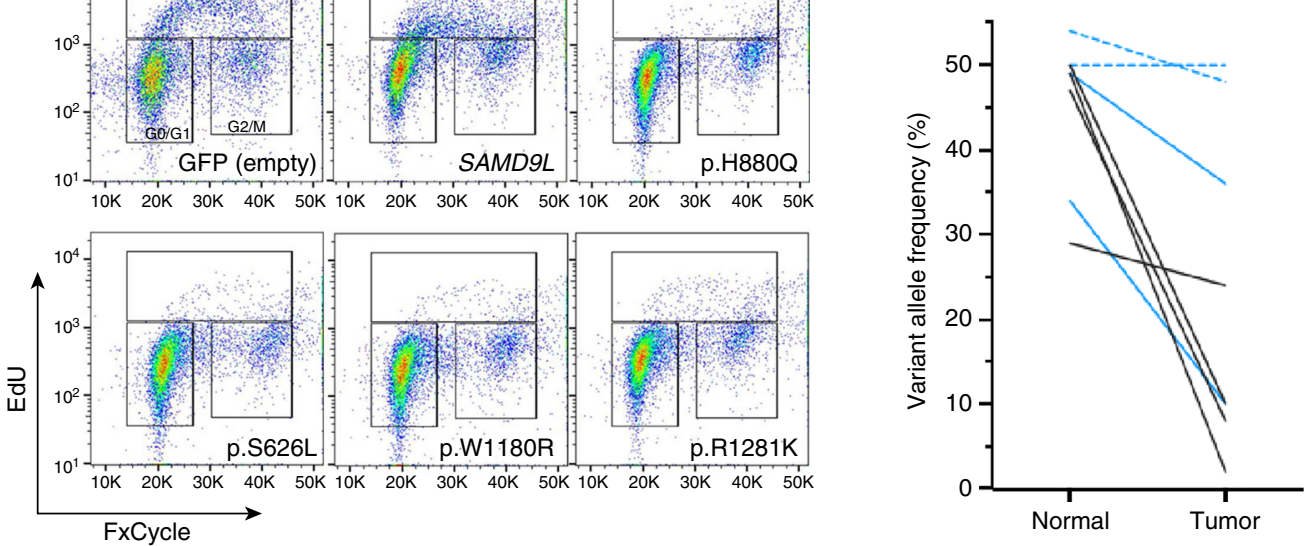

Fig. 4 Gain-of-function mutations in SAMD9 and SAMD9L decrease cell proliferation and inhibit cycle progression. a Schematic showing the protein structure of SAMD9 and SAMD9L. b Flow cytometry plots (FxCycle-total DNA content vs. EdU incorporation) showing the cell cycle inhibiting effects of gain-of-function SAMD9L mutations. SAMD9L p.H880Q (positive control) is a gain-of-function mutation previously reported in Ataxia-Pancytopenia syndrome ${ }^{21}$. c EdU incorporation assay showing that gain-of-function SAMD9/SAMD9L mutations inhibit cells from progressing through the cell cycle as depicted by the relative absence of cells in S-phase. SAMD9 p.R1293W (positive control) is a previously reported gain-of-function mutation in MIRAGE syndrome $^{19}$. SAMD9 p.D881G is a common SNP that is not predicted to be pathogenic. ${ }^{\star \star}: p<0.01,{ }^{\star \star \star}: p<0.001,{ }^{\star \star \star \star}: p<0.0001$ (student's $t$-test). Error bars indicate standard deviation. Data in B \& C are representative of 2-3 experiments completed in triplicate. d VAF plot, obtained from targeted deep sequencing, showing the preferential loss (decrease in VAF) of the SAMD9/SAMD9L mutations in the tumor population. Black lines: SAMD9; Blue lines: SAMD9L; dashed lines indicate cases where a subclonal (3/20 metaphases) del(7) was detected only by conventional karyotyping

our cohort, most notably PTPN11 (15 mutations in 14 patients) and NRAS (10 mutations in 9 patients) (Fig. 5a, Supplementary Datas \#3, 6, and 9). In addition to the genes commonly mutated in myeloid malignancies (e.g., NRAS, KRAS, NF1, CBL, and $P T P N 11)$, we also identified mutations in RRAS (germline), $B R A F$, and SOS1 (Supplementary Fig. 10). In total, we identified Ras/MAPK mutations in $55 \%$ of the total cohort and $43 \%$ of primary MDS cases with VAF's ranging from 2 to 85\% (Supplementary Fig. 11). Interestingly, Ras/MAPK mutations were enriched in the higher-grade primary MDS, RAEB (RAEB: $65 \%$ vs RCC: $17 \%, p=0.002$, Fisher's exact test). In particular for PTPN11, the variant was frequently detected in the lymphocyte "normal" sample, suggesting that these are a result of tumor-innormal contamination, or variants that are either germline or mosaic (Supplementary Fig. 12). Due to low number of somatic coding mutations in these cases it is difficult to confidently make this distinction. This pattern was not limited to JMML cases. The BRAF mutations found in our MDS cohort (p.G469A and p.D594N) induce some level of constitutive ERK phosphorylation and confer IL3 independence in the murine $\mathrm{Ba} / \mathrm{F} 3$ cell line $\mathrm{e}^{37}$ (Fig. 5b, c, and Supplementary Fig. 13).

Additional somatic mutations in pediatric MDS. In addition to mutations in the Ras/MAPK pathway, we also observed somatic mutations in SETBP1. As previously reported, these mutations were present in both the JMML cohort ${ }^{30}$ and MDS cohort ${ }^{38}$ CEBPA $(n=1,1 \%)$, RUNX1 $(n=2,3 \%)$, and ETV6 $(n=4,5 \%)$ somatic mutations were also identified. Somatic TP53 mutations, all within the DNA-binding domain, were present in three cases (4\%). DNMT3A, ASXL1, and TET2 mutations were notably absent from our cohort. In contrast to adult MDS ${ }^{5}$, RNA splicing genes (SRSF2: $n=1, U 2 A F 2: n=1, U 2 A F 1: n=1)$ were rarely mutated in our cohort $(n=3,4 \%)$ (Fig. 2c and Supplementary Fig. 14). Collectively, our comprehensive somatic and germline sequencing illustrates new patterns of mutations in pediatric primary MDS (Fig. 6) and highlights differences between MDS/ MPN and AML-MRC (Supplementary Figs. 15 and 16). Within the primary MDS cohort, the presence of Ras/MAPK mutations, monosomy 7 or germline SAMD9/SAMD9L variants do not impact patient outcome (Supplementary Fig. 17). Consistent with other studies, the presence of SETBP1 mutations did appear to be associated with inferior outcome despite small numbers of patients $^{39}$ (Supplementary Fig. 17f).

Fusion events are rare in pediatric MDS. RNA-sequencing of high-quality RNA material from 43 cases demonstrated that fusion events are rare in pediatric MDS. Only 2 fusions (RUNX1MECOM and CSNK1A1-LECT2) were identified in 25 primary MDS patients (Supplementary Fig. 18). RUNX1-MECOM is a known fusion in AML/MDS ${ }^{40}$. The CSNK1A1-LECT2 fusion is 
a

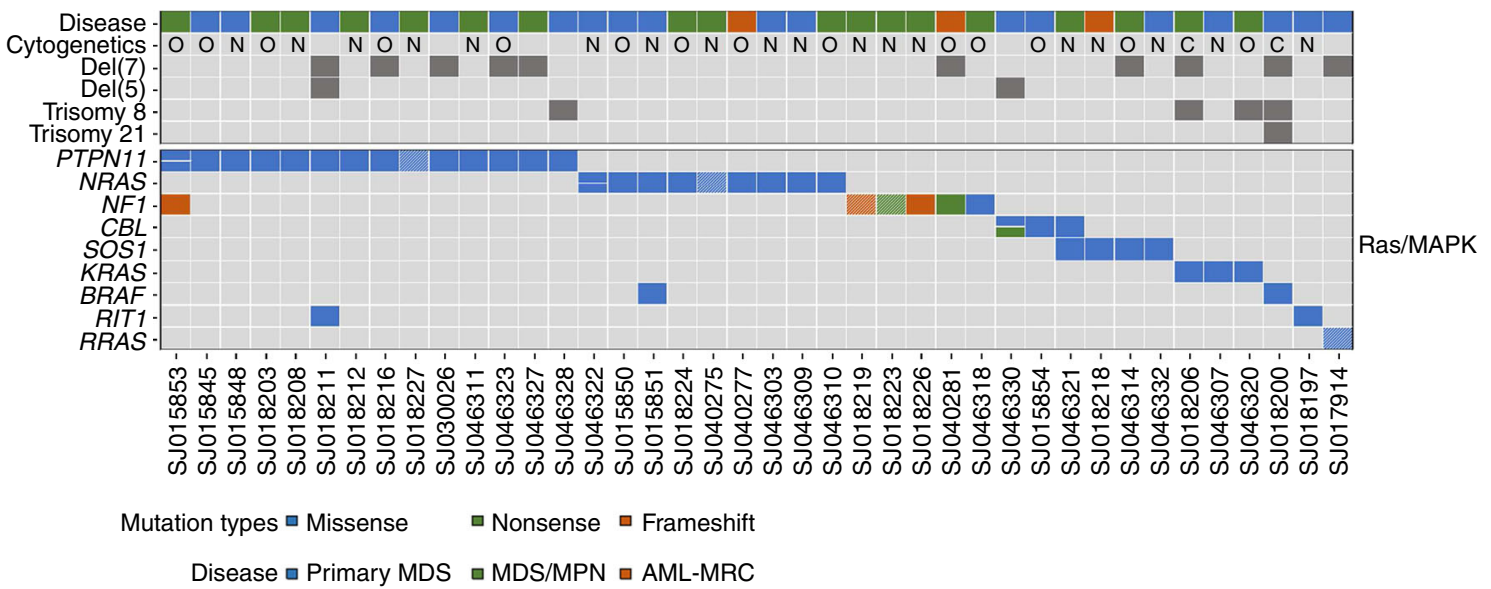

b

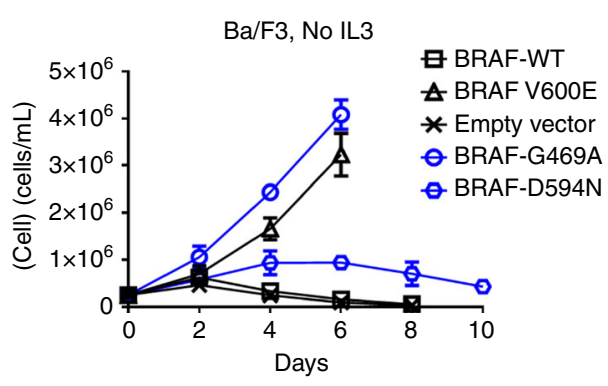

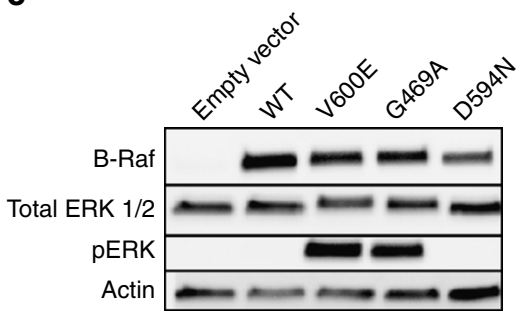

Fig. 5 Ras/MAPK pathway mutations in pediatric MDS, MDS/MPN, and AML-MRC. a Heat map showing all Ras/MAPK pathway mutations, both somatic and presumed germline (cells with hatched lines indicate presumed germline variants from WES tumor/normal cases), in the pediatric MDS cohort $(n=77)$. b Growth curves of $\mathrm{Ba} / \mathrm{F} 3$ cells transduced with retrovirus containing BRAF mutations. Blue curves indicate mutations found in the pediatric MDS cohort, and black curves are positive (V600E) and negative controls. Error bars indicate standard deviation. c Western blots of BRAF, total ERK, and phosphorylated ERK from lysates of 293 T cells transiently transfected with each BRAF mutation. Data are representative of three biological replicates

\section{Table 2 Presumed germline mutations linked to disease}

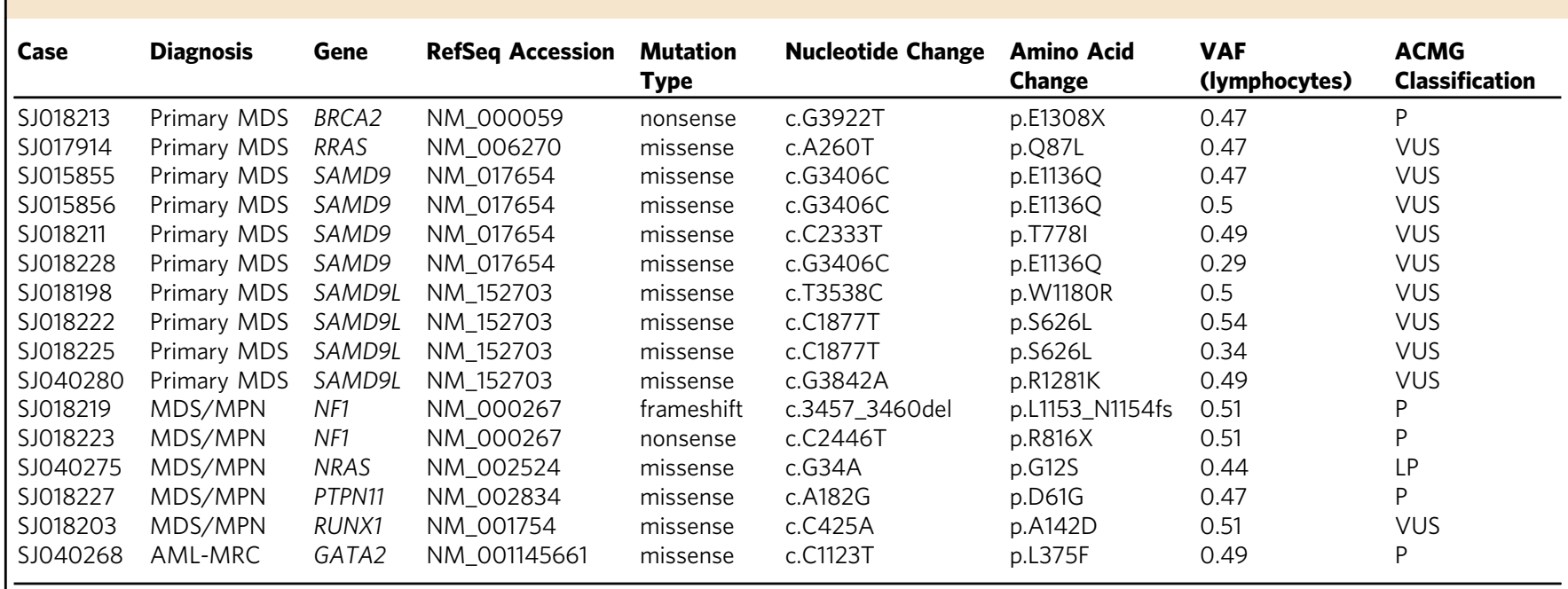

$V A F$, variant allele frequency; $P$ pathogenic; $L P$, likely pathogenic; VUS, variant of unknown significance

Includes only mutations identified by WES in 54 tumor/normal pairs. Note: the VAFs for SJ018228 and SJ018225 are $<40 \%$ because a LOH event is present

the result of an intrachromosomal deletion of chromosome 5 and results in a fusion transcript that removes the kinase domain of CSNK1A1, and is likely similar to other previously reported loss of function alterations in $C S N K 1 A 1^{41}$. Two presumed novel fusion transcripts (NUP98-JADE2 and SNRNP70-FGFR1) were identified in patients with MDS/MPN. JADE2 contains two Plant
Homeo-Domains (PHD), which are common features for translocation partners with $N U P 98^{42}$, and thus is predicated to function similar to NUP98-PHD fusions, like NUP98-NSD1 and NUP98-KDM5A ${ }^{43}$. Likewise, the SNRNP70-FGFR1 preserves the kinase domain of FGFR1, like other FGFR1 translocations in cancer $^{44}$. Four fusion events were detected and validated for 
a
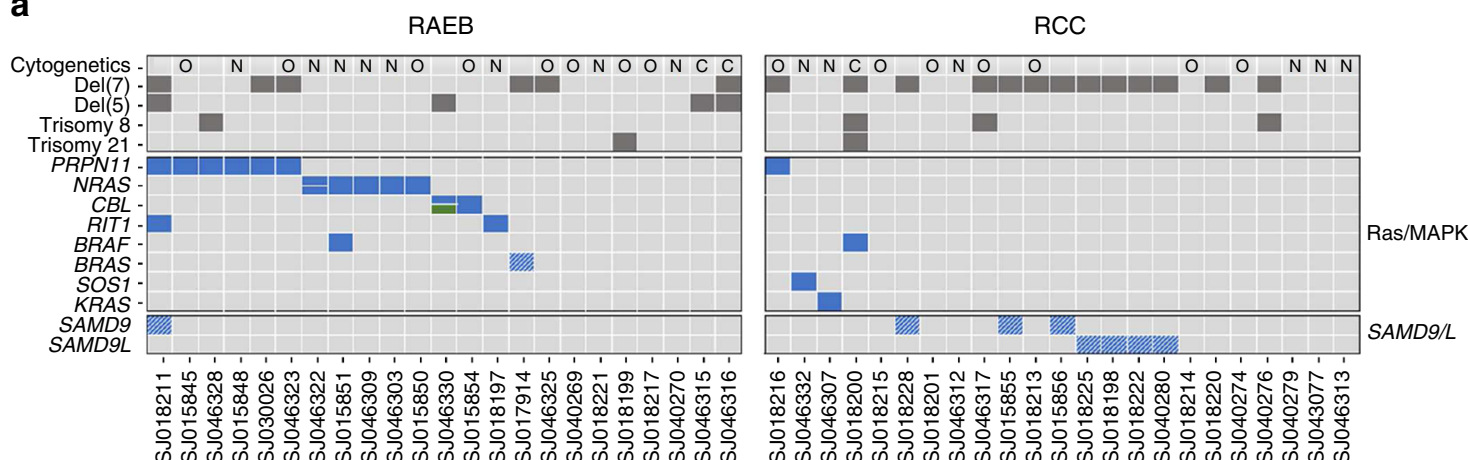

Mutation types $\square$ Missense $\square$ Nonsense

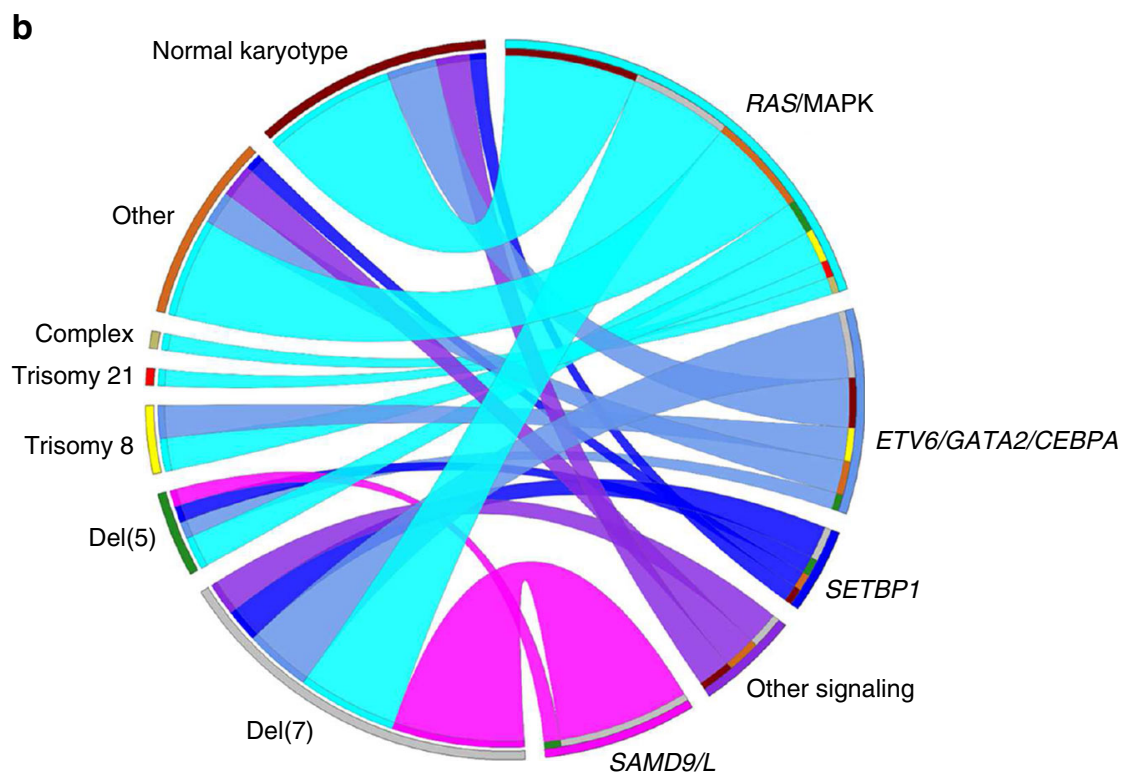

Fig. 6 The genomic landscape of pediatric primary MDS. a Heat map indicating primary MDS patients, subdivided into RCC and RAEB categories, with somatic mutations, germline variants (cells with hatched lines), and transcript fusions. Ras/MAPK mutations are enriched in the RAEB subgroup (65\% vs $17 \%, p=0.002$, Fisher's exact test) b Ribbon plot showing associations between cytogenetic abnormalities and recurrent mutations in myeloid neoplasms. Data from WES and targeted amplicon sequencing of the primary MDS cohort $(n=46)$ was used to build the plot. Associations between a cytogenetic abnormality and a mutation are connected by a ribbon, the width of which is proportional to the frequency of that association

AML-MRC. With the exception of an NF1-RHOT1 fusion that would result in NF1 loss-of-function, the remaining 3 fusions identified (NUP98-KDM5A, DEK-NUP214, ETV6-MECOM) are known to be associated with myeloid malignancies (Supplementary Fig. 19).

\section{Discussion}

Previous studies on pediatric MDS have focused mostly on gene panels ${ }^{31,38}$, which have been guided based on our understanding of adult MDS and inherited bone marrow failure syndromes. In this study we sought to more comprehensively define genetic variation in pediatric MDS through a combination of WES, amplicon sequencing, and RNA-sequencing and our data show a complex pattern of germline and somatic mutations (Fig. 6). We also expanded our analyses to include other pediatric myeloid neoplasms with potentially overlapping morphologic (e.g., dysplasia) or cytogenetic (e.g., del $(7) /(7 q)$ ) features, such as juvenile myelomonocytic leukemia $(n=19)$, unclassifiable myelodysplastic/myeloproliferative neoplasms $(n=4)$, and acute myeloid leukemia with myelodysplasia-related changes $(n=8)$.

We identified germline variants in SAMD9 or SAMD9L in 8 of the 46 cases of primary MDS. SAMD9 and SAMD9L are sterile acid domain containing genes located at $7 \mathrm{q} 21^{45}$, in a region frequently deleted in myeloid tumors ${ }^{46,47}$, and their protein products have been implicated in endosomal function and interferon signaling ${ }^{22,48}$. Loss of murine Samd9l can lead to a MDS-like disease in mice ${ }^{49}$. Recently, germline variants in these genes have been associated with multisystem disorders with a range of systemic abnormalities, including neurologic, endocrine, gastrointestinal, immune, and hematopoietic systems ${ }^{19,21,22}$. A subset of these patients also developed MDS with monosomy 7. It has been proposed that loss of chromosome 7 is a cellular adaptation to the growth suppressive properties of the mutant SAMD9 or SAMD9L protein-referred to previously as "adaptation by aneuploidy" 19 . In all cases, we similarly see selective loss of the chromosome that harbors the heterozygous germline variant. The patients included in this study lack the profound extrahematopoietic phenotypes of MIRAGE and Ataxia-Pancytopenia patients, rather they appear to have isolated MDS. Seven of the 8 patients presented with RCC and had few somatic cooperating events (total somatic mutations: 26, range: 0-10/patient), including 2 cases with no somatic coding mutations. The one patient with RAEB had multiple somatic events, including mutations in PTPN11, ETV6, SETBP1, and deletion of 
chromosome 5 (resulting in the previously mentioned CSNK1A1LECT2 fusion). Although our findings here clearly need to be expanded to a larger cohort, we suggest that germline variants in SAMD9 and SAMD9L are a new class of lesions that need to be screened for in children with MDS and monosomy 7.

In total from the cases with tumor and normal material, we identified 10 primary MDS cases (out of 32) with germline variants that may be causative (Table 2). Interestingly, none of these variants were in GATA2, although in 14 primary MDS cases with tumor-only material available there were 3 additional GATA2 variants ( 1 case with 2 separate mutations) with VAF's $>40 \%$ suggesting that they may be germline events. One AML-MRC patient had a germline GATA2 variant. Five MDS/MPN patients, all JMML, had germline variants, 4 of which were in genes of the Ras/MAPK pathway. Surprisingly, we did not find any germline variants in genes implicated in bone marrow failure syndromes that have overlap with MDS, such as dyskeratosis congenita ${ }^{12,50}$. Of note, our collective findings do show differences in the genomic profiles of pediatric MDS from our single institution cohort and those published by other groups. In addition to fewer germline GATA2 variants, we also observed fewer somatic SETBP1 mutations. We suggest these differences are largely due to the heterogeneous nature of MDS coupled with small cohort sizes.

Monosomy 7 is a known cytogenetic abnormality in many myeloid tumors, and previous studies have demonstrated that it occurs in nearly $25 \%$ of children with MDS $^{15,51}$. The presence of monosomy 7 has also been associated with somatic mutations in $S E T B P 1^{38}$ and germline alterations in GATA2 ${ }^{15}$. Our analyses confirmed the high frequency of deletions involving all or portions of chromosome 7 . In addition, we also observed an association between deletions involving chromosome 7 and two distinct groups (Fig. 6b). In particular, $100 \%$ of patients with SAMD9/SAMD9L mutations (7/8 were classified as RCC), and $71 \%$ of RAEB patients with a Ras/MAPK mutation were associated with chromosome 7 deletions. Clearly, larger studies will be needed to confirm these associations.

We further demonstrated that mutations in the Ras/MAPK pathway are more common in pediatric MDS than adult MDS, especially in children with RAEB. Makishima et al. recently reported WES data for 124 adult MDS patients and Ras/MAPK mutations were present in only $10 \%$ of those cases ${ }^{7}$ vs. our $45 \%$ of pediatric primary MDS. Additionally, the mean mutation frequency per patient (pediatric: 5/case vs. adult: 11.4/case) and the frequency of deletions involving chromosome 5 (pediatric: $5 \%$ vs. adult: $18 \%$ ) were different. These differences are not surprising given the disparate morphologic and clinical characteristics of pediatric and adult MDS. Our data are consistent with the recent study by Pastor et al., but due to the more comprehensive sequencing in our study, we identified mutations in other Ras/ MAPK pathway genes that may not be included in typical panels for genomic testing ${ }^{38}$. Germline and somatic Ras/MAPK mutations largely define JMML $\mathrm{ML}^{24,30,52,53}$ and our data suggest a higher degree of genomic similarity between JMML and pediatric RAEB than previously appreciated, which could have beneficial clinical implications given the new clinical trial with a MEK inhibitor in JMML (Children's Oncology Group ADVL1521). Pediatric MDS is not only contrasted with adult MDS or chronic myelomonocytic leukemia (CMML) in regard to Ras/MAPK pathway mutations but also in mutations of genes encoding epigenetic regulators (more frequent in adult MDS/CMML), thus potentially suggesting that the epigenetic landscape of pediatric MDS is more permissive to transformation than adults.

In summary, we provide the first comprehensive view on the genomic landscape of pediatric MDS. In addition to increasing the spectrum of somatic mutations in pediatric MDS, some of which suggest genomic similarity to JMML, we have expanded the list of genes with potential germline variation in children with MDS. In particular, we define SAMD9 and SAMD9L as new genes linked to childhood MDS.

\section{Methods}

Patient sample details. Tumor and germline samples, when applicable, were obtained with informed consent using a protocol approved by the St. Jude Children's Research Hospital Institutional Review Board. All patients with a diagnosis of MDS, MDS/MPN, and AML-MRC between 1988 and 2016 were originally evaluated for sample adequacy. Diagnoses were reviewed by a hematopathologist (J.M.K.) and classified according to WHO 2008 criteria $^{54}$. Detailed clinicopathological information is available in Supplementary Data \#1. Samples were de-identified before nucleic acid extraction and analysis. The study cohort comprises 46 primary MDS (23 refractory cytopenia of childhood/RCC and 23 refractory anemia with excess blasts/RAEB), 23 MDS/MPN (including 19 with juvenile myelomonocytic leukemia/JMML), and 8 AML-MRC cases for a total cohort of 77 patients. Germline samples were obtained from flow sorted total lymphocytes or CD3 + T-cells from the diagnostic bone marrow samples (Supplementary Fig. 2). Cryopreserved bulk bone marrow cells were thawed in a $37^{\circ} \mathrm{C}$ water bath and transferred to $20 \%$ FBS in PBS to remove residual DMSO according to standard approaches ${ }^{55}$. Cells were lysed with ACK lysing buffer (ThermoFisher A1049201) and washed with PBS prior to staining. The following antibodies were used to immunophenotype the cells and facilitate flow sorting of myeloid and lymphoid populations: CD15-FITC (eBioscience, clone HI98), CD71-BV711 (BD Biosciences, clone M-A712), CD34 PE (Beckman, clones QBEnd10, Immu133, Immu409), CD45R-PerCP-Cy5.5 (eBioscience, clone RA3-6B2), CD235a-PE-Cy7 (BD Biosciences, clone GA-R2), CD3-APC-Cy7 (BD Biosciences, clone SK7), CD33-APC (eBioscience, clone WM-53).

Whole-exome and RNA-sequencing and analysis. DNA and RNA material was isolated from bulk myeloid or isolated lymphocytes by standard phenol:chloroform extraction and ethanol precipitation. Whole-exome sequencing was completed using the Nextera Rapid Capture Expanded Exome reagent (Illumina) and analyzed as previously described ${ }^{56}$. RNA-sequencing was performed using TruSeq Stranded Total RNA library kit (Illumina) and analyzed, as previously described ${ }^{56}$. Structural variation detection was carried out using CICERO $^{57}$, a novel algorithm that uses de novo assembly to identify structural variation in RNA-seq data and Chimerascan $^{58}$. All identified fusions were validated by RT-PCR. Mapping statistics and coverage data are described in Supplementary Data \#2. Recurrent SNVs identified via WES were validated by custom amplicon sequencing using the MiSeq platform as previously described ${ }^{56}$ (Supplementary Data \#12). All SNVs identified by WES and subsequently validated are summarized in Supplementary Data \#3. For the 23 cases without a matched normal sample, DNA from whole bone marrow was analyzed using a TruSeq Custom Amplicon (Illumina) approach (Supplementary Data \#4 for gene/target list). VarScan $2^{59}$ was used for variant calling on the TruSeq Custom Amplicon data with the following criteria: MAPQ $>=1$; minimum read depth at a position to make a call $>=100$; minimum supporting reads at a position to call variants $>=10$; minimum base quality at a position to count a read $>=23$; VAF $>=0.02$. The calls with reads showing strong bias or present in majority of the samples were filtered out, and the remaining ones were manually reviewed. The targeted sites (Supplementary Data \#4) were also scanned regardless of the above cutoffs for manual review. Further, the coding regions for $S A M D 9$ and $S A M D 9 L$ were sequenced using a modified $16 \mathrm{~S}$ library protocol. Short amplicons were used to validate the known variants. Oligonucleotides were designed to amplify an $\sim 350$ bp fragment surrounding each variant and these oligonucleotides included the following Illumina adapters: TCGTCGGCAGCGTCAGATGTGTATAAGAGACAG-[forward primer] and GTCTCGTGGGCTCGG AGATGTGTATAAGAGACAG-[reverse primer]. The amplicons were then purified with Ampure XP beads, PCR-amplified (five cycles) to attach indices and adapters, followed by an additional purification with Ampure XP beads and quality assessment on a LabChip GX. The samples were run on a MiSeq with a 500cycle nano kit. Discovery sequencing to identify any coding SAMD9 or SAMD9L mutations in cases not subjected to WES was performed by amplifying $\sim 1.5 \mathrm{~kb}$ regions with $>50$ overlapping bases. The amplicons were submitted to the Hartwell Center for Nextera XT library preparation, per the manufacturer's protocol, and sequenced on a MiSeq with a 500 cycle nano kit.

CNA detection using whole-exome sequencing data. Samtools ${ }^{60}$ mpileup command was used to generate an mpileup file from matched normal and tumor BAM files with duplicates removed. VarScan2 was then used to take the mpileup file to call somatic CNAs after adjusting for normal/tumor sample read coverage depth and GC content. Circular Binary Segmentation algorithm ${ }^{61}$ implemented in the DNAcopy R package was used to identify the candidate CNAs for each sample. B-allele frequency info for all high quality dbSNPs heterozygous in the germline sample was also used to assess allele imbalance. 
Germline analysis. Whole exome sequencing (WES) data were analyzed using internal workflow that were previously described ${ }^{29}$. Briefly, the sequencing data were analyzed for the presence of single-nucleotide variants and small insertion and deletions and for evidence of germline mosaicism. Germline copy-number variations and structural variations were identified with the use of the Copy Number Segmentation by Regression Tree in Next Generation Sequencing (CONSERTING) $^{62}$ and Clipping Reveals Structure (CREST) ${ }^{63}$ algorithms. For all SNPs and INDELs, functional prediction (e.g., SIFT, CADD, and Polyphen) and population minor allele frequency (MAF) were annotated. In this work, 3 databases were used for population MAF annotation: (i) NHLBI GO Exome Sequencing Project (http://evs.gs.washington.edu/EVS/); (ii) 1000 genomes (http://www. internationalgenome.org); and (iii) ExAC non-TCGA version (http://exac. broadinstitute.org/). A gene list of 1176 genes were composed from various resources: (i) literature review of genes that are potentially involved in AML, MDS, inherited bone marrow failure syndromes, as well as other cancer types ${ }^{16,29,31,32,64}$ (ii) genes that were involved in splicing from predefined pathways (e.g., splicing) in KEGG, GeneOntology, Reactome, Gene Set Enrichment Analysis (GSEA), and NCBI (Supplementary Data \#6). Novel or extremely rare variants $(\mathrm{MAF} \leq 0.1 \%$ ) that passed sequence quality check were kept for subsequent analysis. All nonsynonymous mutations in 207 of these genes were comprehensively reviewed and classified according to ACMG guidelines ${ }^{33}$ by a medical geneticist (C.K.). Nonsynonymous mutations in the remaining 969 genes were merely reported (Supplementary Data \#9). In addition, nonsense, frameshift, or splicing mutations in all other genes covered by the exome capture were also reported (Supplementary Data \#10). Relevant germline variants were validated by targeted resequencing.

Cloning and mutagenesis. pBABE-bleo[BRAFV600E] was a gift from Christopher Counter via Addgene (\#53156). BRAF p.V600E was reverted back to wild-type (NM_004333) using the GENEART ${ }^{\circledR}$ Site Directed Mutagenesis System (Invitrogen). The patient specific mutations found in our MDS cohort were then introduced into the wild-type BRAF containing vector. The pCMV6-Entry[SAMD9] (RC219076) and pCMV6-Entry[SAMD9L] (RC207886) vectors were purchased from Origene. SAMD9 and SAMD9L were PCR amplified such that the resultant amplicons contained a $5^{\prime}$-CACC overhang to allow for direct cloning into the Gateway entry vector, pENTR/D-TOPO. SAMD9/L GFP fusions were created by using the Gateway LR clonase II reaction with the pcDNA6.2/N-EmGFP-DEST Gateway destination vector which was purchased from Lifetech (V35620). The pcDNA6.2/N-EmGFP/GW/CAT vector was used as the GFP-empty vector control. Mutagenesis and PCR amplification primers mentioned above are listed in Supplementary Data \#13.

Cell culture, transient transfection, \& stable transduction. For $B R A F$ functional assays murine-specific retrovirus containing $B R A F$ constructs was produced via transient transfection (FuGene ${ }^{\circledast}$, Promega) of HEK-293T cells (ATCC) with the construct of interest and the EcoPak viral packaging vector, according to standard procedures ${ }^{65}$. Briefly, $48 \mathrm{~h}$ following transfection $\mathrm{Ba} / \mathrm{F} 3$ cells were transduced with viral particles via spinfection/polybrene. For spinfection, $1.5 \mathrm{~mL}$ of virus particle containing supernatant from $293 \mathrm{~T}$ culture was mixed with $\sim 2 \times 10^{6} \mathrm{Ba} / \mathrm{F} 3$ cells in a total volume of $3 \mathrm{~mL}$ of media containing HEPES buffer and $2 \mathrm{uL}$ of polybrene. Cells and virus were then centrifuged at $2000 \times \mathrm{g}$ at $30^{\circ} \mathrm{C}$ for $90 \mathrm{~min}$, after which they were allowed to rest at $37^{\circ} \mathrm{C}, 5 \% \mathrm{CO}_{2}$ for an additional $90 \mathrm{~min}$. Following the rest period, the cells were washed with PBS, centrifuged, and suspended in fresh media (RPMI 1640, 10\% FBS). After $48 \mathrm{~h}$ of transduction Ba/F3 cells were counted via hemacytometer and trypan blue exclusion every 2 days. For SAMD9/SAMD9L functional assays $293 \mathrm{~T}$ cells were transiently transfected with constructs of interest (FuGene ${ }^{\circledR}$, Promega).

Dye dilution cell proliferation assays. Prior to $S A M D 9 / S A M D 9 L$ transfection 293 $\mathrm{T}$ cells were stained with either CellTrace ${ }^{\mathrm{m}}$ Violet (for dye dilution experiment only) or CellTrace $e^{\text {mit }}$ Yellow (for combination dye dilution and EdU incorporation cell cycle assays) (Invitrogen) according to the manufacturer's protocol. In total, $72 \mathrm{~h}$ following transfection $293 \mathrm{~T}$ cells were collected, fixed with $4 \%$ paraformaldehyde, and analyzed by flow cytometry.

EdU cell cycle assays. Following a $24-48 \mathrm{~h}$ transfection with SAMD9/L 293 $\mathrm{T}$ cells were treated with $10 \mathrm{uM}$ EdU for $2 \mathrm{~h}$. Following the EdU incubation, 293 $\mathrm{T}$ cells were harvested and fixed with $4 \%$ paraformaldehyde. After fixing, the ClickIt ${ }^{\circledR}$ reaction (Invitrogen, C10635) was performed according to the manufacturer's protocol. Following the Click-It ${ }^{\circledR}$ reaction, total DNA was labeled with FxCycle (Invitrogen, F10347), and analyzed by flow cytometry.

Immunoblotting and serum stimulation. For BRAF studies, $48 \mathrm{hrs}$ following viral transduction, Ba/F3 cell lysates were prepared with Laemmli buffer and separated on standard polyacrylamide gels. For SAMD9/SAMD9L studies, the induction of phosphorylated ERK was assayed following serum stimulation. Twenty-four $\mathrm{h}$ after SAMD $/ L$ transfection, $293 \mathrm{~T}$ media (10\% FBS) was replaced with serum deficient (1\% FBS) media for 18-24 h. Subsequently, cell lysates were prepared with Laemmli buffer at three time points: 0 min (prior to $10 \%$ FBS replacement) and 10 and $60 \mathrm{~min}$ following full media replacement. The following antibodies were used at a 1:1000 dilution for immunoblotting: SAMD9 (abcam, ab180575), GFP (Invitrogen, A11122), BRAF (Santa Cruz Biotechnology, sc-5284), and from Cell Signaling Technologies: Total ERK (4695S), Phos-ERK (9101S), and GAPDH (2118S).

Statistical methods. The student's $t$-test, two-tailed, assuming equal variances, was used when comparing two experimental groups (e.g., SAMD9/SAMD9L mutations) or diagnostic subgroups (e.g., mutation frequency). The Fisher's exact test was used to compare the frequency of Ras/MAPK mutations between RAEB and RCC subgroups. Overall survival was defined as the time difference between the date of MDS, MDS/MPN, or AML-MRC diagnosis and the date of death. Patients who were alive at the time of last follow up were considered censored. Survival curves between groups were compared via log-rank tests.

Data availability and accession codes. Genomic data have been deposited in the European Genome-phenome Archive (EGA), which is hosted by the European Bioinformatics Institute (EBI), under accession EGAS00001002202. All other remaining data are available within the Article and Supplementary Files, or available from the authors upon request.

Received: 18 May 2017 Accepted: 29 September 2017 Published online: 16 November 2017

\section{References}

1. Glaubach, T., Robinson, L. J. \& Corey, S. J. Pediatric myelodysplastic syndromes: they do exist! J. Pediatr. Hematol. Oncol. 36, 1-7 (2014).

2. Hasle, H. Myelodysplastic and myeloproliferative disorders of childhood. Hematol. Am. Soc. Hematol. Educ. Program 2016, 598-604 (2016).

3. Papaemmanuil, E. et al. Somatic SF3B1 mutation in myelodysplasia with ring sideroblasts. N. Engl. J. Med. 365, 1384-1395 (2011).

4. Graubert, T. A. et al. Recurrent mutations in the U2AF1 splicing factor in myelodysplastic syndromes. Nat. Genet. 44, 53-57 (2011).

5. Haferlach, T. et al. Landscape of genetic lesions in 944 patients with myelodysplastic syndromes. Leukemia 28, 241-247 (2014).

6. Yoshida, K. et al. Frequent pathway mutations of splicing machinery in myelodysplasia. Nature 478, 64-69 (2011).

7. Makishima, H. et al. Dynamics of clonal evolution in myelodysplastic syndromes. Nat. Genet. 49, 204-212 (2017).

8. Hirabayashi, S. et al. Spliceosomal gene aberrations are rare, coexist with oncogenic mutations, and are unlikely to exert a driver effect in childhood MDS and JMML. Blood 119, e96-e99 (2012).

9. Lindsley, R. C. et al. Prognostic mutations in myelodysplastic syndrome after stem-cell transplantation. N. Engl. J. Med. 376, 536-547 (2017).

10. Arber, D. A. et al. The 2016 revision to the World Health Organization classification of myeloid neoplasms and acute leukemia. Blood 127, 2391-2405 (2016).

11. Zhang, M. Y. et al. Germline ETV6 mutations in familial thrombocytopenia and hematologic malignancy. Nat. Genet. 47, 180-185 (2015).

12. Babushok, D. V., Bessler, M. \& Olson, T. S. Genetic predisposition to myelodysplastic syndrome and acute myeloid leukemia in children and young adults. Leuk. Lymphoma 57, 520-536 (2016).

13. Song, W. J. et al. Haploinsufficiency of CBFA2 causes familial thrombocytopenia with propensity to develop acute myelogenous leukaemia. Nat. Genet. 23, 166-175 (1999).

14. Smith, M. L., Cavenagh, J. D., Lister, T. A. \& Fitzgibbon, J. Mutation of CEBPA in familial acute myeloid leukemia. N. Engl. J. Med. 351, 2403-2407 (2004).

15. Wlodarski, M. W. et al. Prevalence, clinical characteristics, and prognosis of GATA2-related myelodysplastic syndromes in children and adolescents. Blood 127, 1387-1397 (2016)

16. Zhang, M. Y. et al. Genomic analysis of bone marrow failure and myelodysplastic syndromes reveals phenotypic and diagnostic complexity. Haematologica 100, 42-48 (2015).

17. Noris, P. et al. ANKRD26-related thrombocytopenia and myeloid malignancies. Blood 122, 1987-1989 (2013).

18. Kirwan, M. et al. Exome sequencing identifies autosomal-dominant SRP72 mutations associated with familial aplasia and myelodysplasia. Am. J. Hum. Genet. 90, 888-892 (2012).

19. Narumi, S. et al. SAMD9 mutations cause a novel multisystem disorder, MIRAGE syndrome, and are associated with loss of chromosome 7. Nat. Genet 48, 792-797 (2016).

20. Buonocore, F. et al. Somatic mutations and progressive monosomy modify SAMD9-related phenotypes in humans. J. Clin. Invest. 127, 1700-1713 (2017).

21. Chen, D. H. et al. Ataxia-pancytopenia syndrome is caused by missense mutations in SAMD9L. Am. J. Hum. Genet. 98, 1146-1158 (2016).

22. Tesi, B. et al. Gain-of-function SAMD9L mutations cause a syndrome of cytopenia, immunodeficiency, MDS and neurological symptoms. Blood 129, 2266-2279 (2017). 
23. Schwartz, J. et al. Germline SAMD9 mutation in siblings with monosomy 7 and myelodysplastic syndrome. Leukemia 31, 1827-1830 (2017).

24. Sakaguchi, H. et al. Exome sequencing identifies secondary mutations of SETBP1 and JAK3 in juvenile myelomonocytic leukemia. Nat. Genet. 45, 937-941 (2013)

25. Klco, J. M. et al. Association between mutation clearance after induction therapy and outcomes in acute myeloid leukemia. JAMA 314, 811-822 (2015).

26. Alexandrov, L. B. et al. Signatures of mutational processes in human cancer. Nature 500, 415-421 (2013).

27. Miles, D. K. et al. Patterns of hematopoietic lineage involvement in children with neurofibromatosis type 1 and malignant myeloid disorders. Blood 88, 4314-4320 (1996).

28. Cooper, L. J. et al. Evidence that juvenile myelomonocytic leukemia can arise from a pluripotential stem cell. Blood 96, 2310-2313 (2000).

29. Zhang, J. et al. Germline mutations in predisposition genes in pediatric cancer. N. Engl. J. Med. 373, 2336-2346 (2015).

30. Stieglitz, E. et al. The genomic landscape of juvenile myelomonocytic leukemia. Nat. Genet. 47, 1326-1333 (2015).

31. Keel, S. B. et al. Genetic features of myelodysplastic syndrome and aplastic anemia in pediatric and young adult patients. Haematologica 101, 1343-1350 (2016)

32. Wong, T. N. et al. Role of TP53 mutations in the origin and evolution of therapy-related acute myeloid leukaemia. Nature 518, 552-555 (2015).

33. Richards, S. et al. Standards and guidelines for the interpretation of sequence variants: a joint consensus recommendation of the american college of medical genetics and genomics and the association for molecular pathology. Genet. Med. 17, 405-424 (2015).

34. Suzuki, J., Kaziro, Y. \& Koide, H. An activated mutant of R-Ras inhibits cell death caused by cytokine deprivation in BaF3 cells in the presence of IGF-I. Oncogene 15, 1689-1697 (1997).

35. Saez, R., Chan, A. M., Miki, T. \& Aaronson, S. A. Oncogenic activation of human R-ras by point mutations analogous to those of prototype $\mathrm{H}$-ras oncogenes. Oncogene 9, 2977-2982 (1994).

36. Flex, E. et al. Activating mutations in RRAS underlie a phenotype within the RASopathy spectrum and contribute to leukaemogenesis. Hum. Mol. Genet. 23, 4315-4327 (2014).

37. Warmuth, M., Kim, S., Gu, X. J., Xia, G. \& Adrian, F. Ba/F3 cells and their use in kinase drug discovery. Curr. Opin. Oncol. 19, 55-60 (2007).

38. Pastor, V. et al. Mutational landscape in children with myelodysplastic syndromes is distinct from adults: specific somatic drivers and novel germline variants. Leukemia 31, 759-762 (2017).

39. Stieglitz, E. et al. Subclonal mutations in SETBP1 predict relapse in juvenile myelomonocytic leukemia. Blood 125, 516-524 (2015).

40. Shen, L. et al. RUNX1-Evi-1 fusion gene inhibited differentiation and apoptosis in myelopoiesis: an in vivo study. BMC Cancer. 15, 970 (2015).

41. Bidere, N. et al. Casein kinase lalpha governs antigen-receptor-induced NF-kappaB activation and human lymphoma cell survival. Nature 458, 92-96 (2009).

42. Gough, S. M., Slape, C. I. \& Aplan, P. D. NUP98 gene fusions and hematopoietic malignancies: common themes and new biologic insights. Blood 118, 6247-6257 (2011).

43. Wang, G. G. et al. Haematopoietic malignancies caused by dysregulation of a chromatin-binding PHD finger. Nature 459, 847-851 (2009).

44. Gallo, L. H., Nelson, K. N., Meyer, A. N. \& Donoghue, D. J. Functions of fibroblast growth factor receptors in cancer defined by novel translocations and mutations. Cytokine Growth Factor Rev. 26, 425-449 (2015).

45. Lemos de Matos, A., Liu, J., McFadden, G. \& Esteves, P. J. Evolution and divergence of the mammalian SAMD9/SAMD9L gene family. BMC Evol. Biol. 13, 121 (2013)

46. Asou, H. et al. Identification of a common microdeletion cluster in 7 q21.3 subband among patients with myeloid leukemia and myelodysplastic syndrome. Biochem. Biophys. Res. Commun. 383, 245-251 (2009).

47. Kotini, A. G. et al. Functional analysis of a chromosomal deletion associated with myelodysplastic syndromes using isogenic human induced pluripotent stem cells. Nat. Biotechnol. 33, 646-655 (2015).

48. Pappas, D. J. et al. Longitudinal system-based analysis of transcriptional responses to type I interferons. Physiol. Genomics 38, 362-371 (2009).

49. Nagamachi, A. et al. Haploinsufficiency of SAMD9L, an endosome fusion facilitator, causes myeloid malignancies in mice mimicking human diseases with monosomy 7. Cancer Cell 24, 305-317 (2013).

50. Savage, S. A. Dyskeratosis congenita. In Gene Reviews (eds Pagon, R. A. et al.) (Seattle WA, 1993).

51. Sasaki, H. et al. Myelodysplastic syndrome in childhood: a retrospective study of 189 patients in Japan. Leukemia 15, 1713-1720 (2001).

52. Caye, A. et al. Juvenile myelomonocytic leukemia displays mutations in components of the RAS pathway and the PRC2 network. Nat. Genet. 47, 1334-1340 (2015)

53. Tartaglia, M. et al. Somatic mutations in PTPN11 in juvenile myelomonocytic leukemia, myelodysplastic syndromes and acute myeloid leukemia. Nat. Genet. 34, 148-150 (2003).
54. Vardiman, J. W. et al. The 2008 revision of the World Health Organization (WHO) classification of myeloid neoplasms and acute leukemia: rationale and important changes. Blood 114, 937-951 (2009).

55. Klco, J. M. et al. Genomic impact of transient low-dose decitabine treatment on primary AML cells. Blood 121, 1633-1643 (2013).

56. Faber, Z. J. et al. The genomic landscape of core-binding factor acute myeloid leukemias. Nat. Genet. 48, 1551-1556 (2016).

57. Li, Y. et al. Detecting complex fusion transcripts in pediatric cancer using a novel assembly-based algorithm CICERO (1387M). Presented at the 64th Annual Meeting of the American Society of Human Genetics, October 18-22, San Diego, CA (American Society of Human Genetics, Bethesda, MD, 2014).

58. Iyer, M. K., Chinnaiyan, A. M. \& Maher, C. A. ChimeraScan: a tool for identifying chimeric transcription in sequencing data. Bioinformatics 27, 2903-2904 (2011).

59. Koboldt, D. C. et al. VarScan 2: somatic mutation and copy number alteration discovery in cancer by exome sequencing. Genome Res. 22, 568-576 (2012).

60. Li, H. et al. The Sequence alignment/map format and SAMtools. Bioinformatics 25, 2078-2079 (2009).

61. Olshen, A. B., Venkatraman, E. S., Lucito, R. \& Wigler, M. Circular binary segmentation for the analysis of array-based DNA copy number data. Biostatistics 5, 557-572 (2004).

62. Chen, X. et al. CONSERTING: integrating copy-number analysis with structural-variation detection. Nat. Methods 12, 527-530 (2015).

63. Wang, J. et al. CREST maps somatic structural variation in cancer genomes with base-pair resolution. Nat. Methods 8, 652-654 (2011).

64. Cancer Genome Atlas Research, N. et al. Genomic and epigenomic landscapes of adult de novo acute myeloid leukemia. N. Engl. J. Med. 368, 2059-2074 (2013).

65. Grieselhuber, N. R. et al. Notch signaling in acute promyelocytic leukemia. Leukemia 27, 1548-1557 (2013).

\section{Acknowledgements}

We thank all the patients and their parents at SJCRH for contributing material for this study. We thank the Biorepository, the Flow Cytometry and Cell Sorting Core, and the Hartwell Center for Bioinformatics and Biotechnology of St. Jude Children's Research Hospital. Jinghui Zhang and the Department of Computational Biology graciously provided previously established bioinformatic pipelines and support. We thank Drs. Timothy Ley and Matthew Walter for critical review of this manuscript. This work was funded by the American Lebanese and Syrian Associated Charities of St. Jude Children's Research Hospital and grants from the US National Institutes of Health (P30 CA021765, Cancer Center Support Grant, and K08 HL116605 (J.M.K.)). J.M.K. holds a Career Award for Medical Scientists from the Burroughs Wellcome Fund.

\section{Author contributions}

J.R.S., J.M., T.L., and J.M.K. prepared the manuscript. J.R.S., T.L., V.B., and J.M.K. were responsible for experimental design and analysis. T.L. prepared DNA and RNA from all patient samples. J.M., M.W., S.W., G.S., G.W., J.E. were responsible for bioinformatic data analysis. C.K., K.E.N., and J.M.K. analyzed germline variants and determined their likely pathogenicity. C.G.M. and R.C.R. assisted with data analysis and acquisition of patient cases.

\section{Additional information}

Supplementary Information accompanies this paper at doi:10.1038/s41467-017-01590-5.

Competing interests: The authors declare no competing financial interests.

Reprints and permission information is available online at http://npg.nature.com/ reprintsandpermissions/

Publisher's note: Springer Nature remains neutral with regard to jurisdictional claims in published maps and institutional affiliations.

\footnotetext{
Open Access This article is licensed under a Creative Commons Attribution 4.0 International License, which permits use, sharing, adaptation, distribution and reproduction in any medium or format, as long as you give appropriate credit to the original author(s) and the source, provide a link to the Creative Commons license, and indicate if changes were made. The images or other third party material in this article are included in the article's Creative Commons license, unless indicated otherwise in a credit line to the material. If material is not included in the article's Creative Commons license and your intended use is not permitted by statutory regulation or exceeds the permitted use, you will need to obtain permission directly from the copyright holder. To view a copy of this license, visit http://creativecommons.org/ licenses/by/4.0/.
}

(C) The Author(s) 2017 